Article

\title{
Cooperative Non-Orthogonal Multiple Access over Log-Normal Power Line Communication Channels
}

\author{
Roger Kwao Ahiadormey $(\mathbb{D}$, Prince Anokye $(\mathbb{C}$ and Kyoung-Jae Lee * (]) \\ Department of Electronics and Control Engineering, Hanbat National University, Daejeon 34158, Korea; \\ rogerkwao@gmail.com (R.K.A.); princemcanokye@yahoo.com (P.A.) \\ * Correspondence: kyoungjae@hanbat.ac.kr; Tel.: +82-42-821-1730
}

Received: 11 September 2019; Accepted: 15 October 2019; Published: 1 November 2019

\begin{abstract}
In this paper, we analyze the performance of cooperative power domain non-orthogonal multiple access (NOMA) in power line communication (PLC) networks. Due to the high signal attenuation of the source to user links, a relay aids communication from the source to two users. With half-duplex transmission, the source transmits a superimposed symbol in the first phase. The relay utilizes amplify-and-forward (AF) and decode-and-forward (DF) protocol on the received superimposed signal and forwards it to the users in the second phase. We derive analytic expressions for the outage probability and the system throughput of the proposed system under a PLC log-normal channel with impulsive noise. Based on the results for AF NOMA relaying case, we analyze the system performance at high signal-to-noise ratio (SNR) and derive closed-form lower and upper bounds for the outage probability. Simulation results show an improvement in the outage probability and the system throughput performance of the AF and DF NOMA schemes compared to the NOMA without relaying transmission and conventional orthogonal multiple access scheme. Furthermore, the impact of the channel variance is highlighted in the results. It is shown that the DF NOMA has a better outage probability than the AF NOMA scheme for low channel variance scenarios (i.e., less branches and connected loads in the PLC network). However, as the channel variance increases, AF NOMA scheme has similar outage probability performance as the DF NOMA scheme. In addition, it is shown that the system throughput is enhanced when the relay employs DF relaying compared to AF relaying.
\end{abstract}

Keywords: power line communication; non-orthogonal multiple access; amplify-and-forward relaying; decode-and-forward relaying; outage probability; system throughput

\section{Introduction}

There is a growing body of research that shows non-orthogonal multiple access (NOMA) as a promising multiple access technique for next generation communication systems. The basic idea of NOMA is to allow multiple users to share the same time/frequency/space resources. Generally, NOMA can be applied in the power domain or code domain. However, power domain NOMA (PD-NOMA) has gained more traction since it is easily applicable to current systems [1,2]. In PD-NOMA, a source transmits a superimposed signal to different users with appropriate power allocation. Each user is able to recover its desired signal by the application of successive interference cancellation (SIC). Compared to orthogonal multiple access (OMA) techniques such as time-division multiple access (TDMA) and frequency-division multiple access (FDMA), NOMA offers higher spectral efficiency and achievable rate [3]. However, users with better channel conditions naturally benefit more with NOMA than users with worse channel conditions due to increased multiple access interference at their receivers [4].

The advent of NOMA has enabled research on many conventional techniques, one of which is cooperative relaying [5-9]. In cooperative communications, the relay usually operates with 
amplify-and-forward (AF) or decode-and-forward (DF) protocols where the advantage of the AF protocol lies in its low processing cost compared to the DF protocol [9]. However, it is shown that the two protocols generally achieve very similar performance when the relay to user link is unreliable [10]. Cooperative communication as applied to NOMA comes in two forms. First, there is the user relaying with DF protocol where a user (typically with strong channel conditions) acts as a relay to forward information to the user with weak channel conditions [11-13]. On the other hand, there also exists dedicated relay transmission between the source and the users [14-17]. The capacity of user relaying NOMA was analyzed in Reference [11]. To improve on the ergodic sum-rate and outage probability performance in Reference [11], a novel receiver design was introduced in Reference [12], where the destination jointly decodes the received symbols by using maximum ratio combining (MRC) and SIC. The authors of Reference [13] studied user relaying with the capability to switch between half-duplex (HD) and full-duplex (FD) modes to enhance system performance. Under direct and non-direct link scenarios, FD NOMA is shown to be better than HD NOMA in terms of the outage probability and the ergodic sum-rate in the low signal-to-noise ratio (SNR) region. While variable gain AF relaying was studied in Reference [14], the authors of Reference [15] considered a fixed gain AF relay with direct and non-direct links. In References [14,15], the system performance was analyzed under the assumption of Nakagami- $m$ channels. Moreover, a comparison of AF and DF relaying with partial channel state information (CSI) was discussed in Reference [16]. Based on the analysis, it is shown that, although DF relaying has better outage probability than AF relaying, the performance gap between AF and DF relaying for the outage probability is negligible as the SNR increases.

As an enabler of smart grid (SG) and Internet of Things (IoT) applications, power line communication (PLC) is seen as an attractive and promising technique due to the ubiquitous nature of power lines [18]. Naturally, the application of NOMA to PLC can only prove beneficial [19-22]. In Reference [19], user relaying NOMA with DF protocol was proposed for PLC systems. The average sum capacity was analyzed and results show NOMA can significantly improve the performance of PLC compared to OMA and satisfy the electromagnetic compatibility (EMC) requirements [23]. The authors of [20] studied a two-stage NOMA scheme, where NOMA is applied at both the source and the user relay, which is shown to outperform the one-stage system in Reference [19]. An adaptive cooperative NOMA scheme for PLC was proposed in Reference [21], where a dedicated DF relay establishes communication between a source modem and two user modems. Depending on the feedback information in the second phase, a direct or cooperative transmission increases the system throughput performance compared to TDMA and a conventional cooperative NOMA scheme. A joint power allocation was proposed for a multi-user NOMA visbile light communication (VLC) network in Reference [22]. Here, VLC is enabled by a PLC modem. By jointly optimizing the allocated power to the PLC and VLC links, NOMA performs better than OMA in terms of sum throughput. The aforementioned works [19-21] have only considered DF relaying with NOMA in cooperative PLC systems.

In this paper, we propose cooperative NOMA for PLC systems. The relay aids communication between the source and two users (near and far users) due to the high signal attenuation of the direct link. This is in contrast to the system model studied in References [21,24], where a direct link exists between the source and the near user. We study the system model under a log-normal fading assumption with impulsive noise characteristic to PLC networks [25-29]. Data communication is executed in two equal phases. In the first phase of communication, the source modem transmits a superimposed signal with appropriate power allocation to the relay modem. The relay utilizes the AF or DF protocol on the received signal and forwards it to the two users in the second phase. We derive analytic expressions for the outage probability and the system throughput for the AF and DF NOMA protocols. By analyzing the results of the AF NOMA scheme in the high SNR region, we obtain closed-form lower and upper bounds of the outage probability. The derived analytic expressions are shown to be tight in comparison with Monte Carlo simulations. Furthermore, we show that the derived closed-form lower bound is able to approximate the outage probability especially at high SNR. The superiority of the proposed AF and DF NOMA schemes is illustrated by comparing with the 
conventional OMA scheme and direct NOMA transmission without relaying. From the simulation results, it is revealed that the DF NOMA outperforms AF NOMA in terms of outage probability in low channel variance settings. However, as the channel variance increases, DF NOMA has similar performance with the AF NOMA scheme. Furthermore, it is shown that the system throughput is enhanced when the relay employs DF relaying compared to AF relaying.

The rest of the paper is organized as follows: Section 2 describes the system model for the AF and DF NOMA schemes in PLC networks. Analysis of the outage probability and the system throughput is presented in Section 3. In Section 4, we describe two benchmark schemes for comparison. The simulation results and subsequent discussions are presented in Section 5. Finally, Section 6 concludes the paper.

Notation: $f_{X}(\cdot), F_{X}(\cdot)$ and $\bar{F}_{X}(\cdot)$ denote the probability density function (PDF), cumulative distribution function $(\mathrm{CDF})$ and the complementary $\mathrm{CDF}(\mathrm{CCDF})$ of the random variable (RV) $X$, respectively. $Q(\cdot), \operatorname{Pr}\{\cdot\}, \mathbb{E}[\cdot], \min (\cdot)$ and $\max (\cdot)$ denote the Gaussian $Q$ function, the probability, the expectation, the minimum and the maximum operators, respectively.

\section{System Model}

Consider the cooperative PLC network shown in Figure 1, where a source modem $\mathcal{S}$ communicates with two users $\mathcal{N}$ and $\mathcal{F}$ through a relay $\mathcal{R}$ with AF or DF protocol. It is assumed that the direct link between the source and the users is highly attenuated compared to the source to relay and the relay to user links. The two users, $\mathcal{N}$ and $\mathcal{F}$, are designated as the near user and the far user, respectively. In addition, the CSI is assumed to be perfectly known at all receiving modems. The distance-dependent cable attenuation is modeled as $\alpha_{i}=\exp \left(-\left(b_{0}+b_{1} f^{k}\right) d_{i}\right), i \in\{S R, R N, R F\}$ where $d_{i}$ is the distance between the PLC modems, $f$ represents the operating frequency in $\mathrm{MHz}, k$ is the exponent of the attenuation factor, $b_{0}$ and $b_{1}$ are the attenuation constants acquired from measurement data [30].

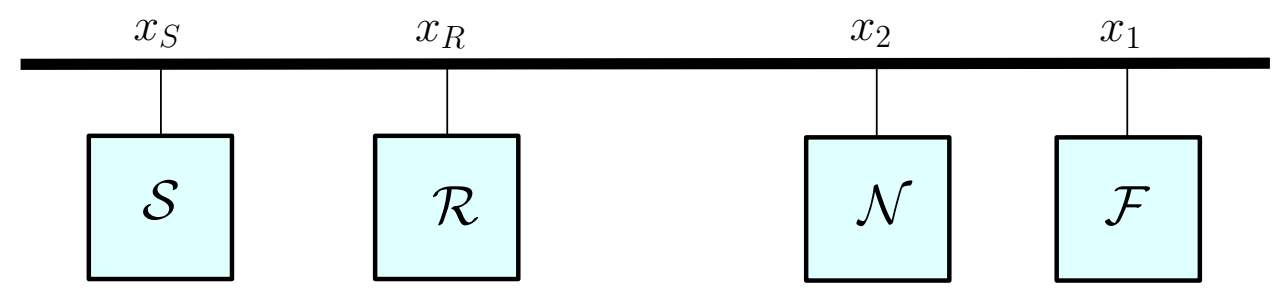

Figure 1. System model of cooperative relaying in power line communication (PLC) networks.

The source-to-relay, relay-to-near user and relay-to-far user channels are denoted by $h_{S R}, h_{R N}$ and $h_{R F}$, respectively. We assume all channels experience independent and identically distributed log-normal fading which is common in the PLC literature [25-27]. The PDF of the PLC log-normal fading channel is given by

$$
f_{h_{i}}(h)=\frac{\xi}{\sqrt{2 \pi} \sigma_{i} h} \exp \left[-\frac{\left(\xi \ln (h)-\mu_{i}\right)^{2}}{2 \sigma_{i}^{2}}\right], h>0,
$$

where $\xi=10 / \ln (10)$ is a scaling constant and $\mu_{i}$ and $\sigma_{i}^{2}$ (in decibels) are the mean and variance of $10 \log _{10}(h)$, respectively, which follows the Gaussian distribution. In PLC networks, the channel variance accounts for the branch network topology where its value increases as the number of branches and connected loads in the network increases [19]. In essence, low channel variance relates to a good fading scenario while high channel variance relates to a bad fading scenario [31].

The performance of any PLC network is limited by the several sources of noise that can be broadly categorized as colored background noise, narrowband interference and impulsive noise [32]. To accurately capture the noise effects, several models have been proposed including the Bernoulli-Gaussian process, Middleton Class A, Markov-Middleton and Markov-Gaussian models $[28,29]$. In this work, we adopt the Bernoulli-Gaussian model due to its mathematical 
tractability [28]. Using the Bernoulli-Gaussian model, the PLC noise is modeled as an aggregate of background noise and impulsive noise [26]. The impulsive noise is assumed to occur with a probability of $p$ while the background noise occurs with a probability of $1-p$ in a transmission block.

The proposed relaying strategy with NOMA for the cooperative PLC network occurs in two phases. Let $x_{1}$ and $x_{2}$ denote the messages to be transmitted with $\mathbb{E}\left[\left|x_{1}\right|^{2}\right]=\mathbb{E}\left[\left|x_{2}\right|^{2}\right]=1$. During the first phase, the source modem $\mathcal{S}$ sends the superimposed signal expressed as $x_{S}=\sqrt{a_{1} P_{S}} x_{1}+\sqrt{a_{2} P_{S}} x_{2}$ to the relay modem $\mathcal{R}$. Here, $P_{S}$ is the source transmit power, $a_{1}$ and $a_{2}$ are the power allocation coefficients for $x_{1}$ and $x_{2}$, respectively. Due to the weak channel conditions of the far user $\mathcal{F}$, its designated symbol $x_{1}$, is allocated more power. Therefore, the following conditions hold: $a_{1}>a_{2}$ and $a_{1}+a_{2}=1$. The received signal at $\mathcal{R}$ is expressed as

$$
y_{R}=\left(\sqrt{a_{1} P_{S}} x_{1}+\sqrt{a_{2} P_{S}} x_{2}\right) \alpha_{S R} h_{S R}+n_{R}
$$

where $n_{R}$ represents the noise at $\mathcal{R}$ with variance $\sigma_{R}^{2}$.

In the second phase, the relay forwards a new data signal $x_{R}$ to the two users after applying the $\mathrm{AF}$ or DF protocol. While the AF protocol amplifies the received signal, the DF protocol rebuilds the superimposed signal of $x_{1}$ and $x_{2}$ upon successful decoding of the received signal [16]. With a relay transmit power $P_{R}$, the transmitted signal $x_{R}$, is expressed as

$$
x_{R}= \begin{cases}\beta \sqrt{P_{R}} y_{R}, & \text { for AF protocol, } \\ \sqrt{a_{1} P_{R}} x_{1}+\sqrt{a_{2} P_{R}} x_{2}, & \text { for DF protocol, }\end{cases}
$$

where $\beta$ is the variable relay gain given by [33]

$$
\beta=\sqrt{\frac{1}{P_{S} \alpha_{S R}^{2} h_{S R}^{2}+\sigma_{R}^{2}}} .
$$

Therefore, the received signals at the near user $\mathcal{N}$ and the far user $\mathcal{F}$ are expressed, respectively, as

$$
y_{N}=\alpha_{R N} h_{R N} x_{R}+n_{N},
$$

and

$$
y_{F}=\alpha_{R F} h_{R F} x_{R}+n_{F}
$$

where $n_{N}$ and $n_{F}$ represent the noise at the near user and the far user with variance $\sigma_{N}^{2}$ and $\sigma_{F}^{2}$, respectively.

\subsection{Amplify-and-Forward Relaying}

Based on the fact that $x_{1}$ is allocated more power, the far user $\mathcal{F}$ decodes its desired signal $x_{1}$ by treating $x_{2}$ as interference. As a result, the post-detection instantaneous signal-to-interferenceplus-noise ratio (SINR) for $x_{1}$ at the far user is written as

$$
\gamma_{F \rightarrow x_{1}}^{A F}=\frac{a_{1} P_{R} P_{S} \alpha_{S R}^{2} \alpha_{R F}^{2} h_{S R}^{2} h_{R F}^{2}}{a_{2} P_{R} P_{S} \alpha_{S R}^{2} \alpha_{R F}^{2} h_{S R}^{2} h_{R F}^{2}+P_{S} \alpha_{S R}^{2} h_{S R}^{2} \sigma_{F}^{2}+P_{R} \alpha_{R F}^{2} h_{R F}^{2} \sigma_{R}^{2}+\sigma_{R}^{2} \sigma_{F}^{2}} .
$$

In order to retrieve its desired signal $x_{2}$, the near user $\mathcal{N}$ decodes $x_{1}$ and removes it through SIC. Consequently, the post-detection SINRs at the near user for $x_{1}$ and $x_{2}$ are, respectively, given by

$$
\gamma_{N \rightarrow x_{1}}^{A F}=\frac{a_{1} P_{R} P_{S} \alpha_{S R}^{2} \alpha_{R N}^{2} h_{S R}^{2} h_{R N}^{2}}{a_{2} P_{R} P_{S} \alpha_{S R}^{2} \alpha_{R N}^{2} h_{S R}^{2} h_{R N}^{2}+P_{S} \alpha_{S R}^{2} h_{S R}^{2} \sigma_{N}^{2}+P_{R} \alpha_{R N}^{2} h_{R N}^{2} \sigma_{R}^{2}+\sigma_{R}^{2} \sigma_{N}^{2}},
$$


and

$$
\gamma_{N \rightarrow x_{2}}^{A F}=\frac{a_{2} P_{R} P_{S} \alpha_{S R}^{2} \alpha_{R N}^{2} h_{S R}^{2} h_{R N}^{2}}{P_{S} \alpha_{S R}^{2} h_{S R}^{2} \sigma_{N}^{2}+P_{R} \alpha_{R N}^{2} h_{R N}^{2} \sigma_{R}^{2}+\sigma_{R}^{2} \sigma_{N}^{2}} .
$$

\subsection{Decode-and-Forward Relaying}

The DF relay decodes the superimposed signal in the first phased based on the NOMA principle that is, $x_{1}$ is decoded first since it is allocated more power. After this, $x_{2}$ is obtained by SIC where $x_{1}$ is reencoded and subtracted from the composite signal. The instantaneous SINRs for detecting $x_{1}$ and $x_{2}$ are, respectively, written as

$$
\gamma_{R \rightarrow x_{1}}^{D F}=\frac{a_{1} P_{S} \alpha_{S R}^{2} h_{S R}^{2}}{a_{2} P_{S} \alpha_{S R}^{2} h_{S R}^{2}+\sigma_{R}^{2}}
$$

and

$$
\gamma_{R \rightarrow x_{2}}^{D F}=\frac{a_{2} P_{S} \alpha_{S R}^{2} h_{S R}^{2}}{\sigma_{R}^{2}} .
$$

The far and near users can recover their desired signals after relay transmission in the second phase. Since more power is allocated to the far user, it decodes its intended data symbol $x_{1}$ directly by treating $x_{2}$ as interference. The instantaneous SINR at the far user, $\mathcal{F}$, for decoding $x_{1}$ is obtained as

$$
\gamma_{F \rightarrow x_{1}}^{D F}=\frac{a_{1} P_{R} \alpha_{R F}^{2} h_{R F}^{2}}{a_{2} P_{R} \alpha_{R F}^{2} h_{R F}^{2}+\sigma_{F}^{2}} .
$$

To decode its desired data symbol $x_{2}$, the near user first decodes $x_{1}$ and applies SIC. The SINR of detecting $x_{1}$ is expressed as

$$
\gamma_{N \rightarrow x_{1}}^{D F}=\frac{a_{1} P_{R} \alpha_{R N}^{2} h_{R N}^{2}}{a_{2} P_{R} \alpha_{R N}^{2} h_{R N}^{2}+\sigma_{N}^{2}} .
$$

Finally, the instantaneous SNR at the near user for detecting $x_{2}$ is given by

$$
\gamma_{N \rightarrow x_{2}}^{D F}=\frac{a_{2} P_{R} \alpha_{R N}^{2} h_{R N}^{2}}{\sigma_{N}^{2}} .
$$

In the next section, we derive analytic expressions for the outage probability and the system throughput for the proposed AF and DF NOMA schemes under PLC log-normal channels with impulsive noise.

\section{Outage Probability Analysis}

Without loss of generality, we define $\sigma^{2}=\sigma_{R}^{2}=\sigma_{N}^{2}=\sigma_{F}^{2}, \rho_{S}=P_{S} / \sigma^{2}$ and $\rho_{R}=P_{R} / \sigma^{2} \cdot \rho_{S}$ and $\rho_{R}$ denote the source and relay transmit SNR. In this work, we assume erasure decoding, where the received signals affected by impulsive noise are discarded in the decoding process [19-21]. An outage occurs when the achievable rate is less than the predefined target rate. Let $R_{1}$ and $R_{2}$ denote the rate thresholds for $x_{1}$ and $x_{2}$, where the outage SNRs for $x_{1}$ and $x_{2}$ are $\psi_{1}=2^{\frac{2 R_{1}}{1-p}}-1$ and $\psi_{2}=2^{\frac{2 R_{2}}{1-p}}-1$, respectively.

\subsection{Amplify-and-Forward NOMA}

First, we analyze the outage probability of the far user $\mathcal{F}$, which is defined as 


$$
\begin{aligned}
P_{\text {out }, F}^{A F} & =\operatorname{Pr}\left\{\gamma_{F \rightarrow x_{1}}^{A F}<\psi_{1}\right\}, \\
& =1-\operatorname{Pr}\left\{\gamma_{F \rightarrow x_{1}}^{A F}>\psi_{1}\right\} .
\end{aligned}
$$

By defining $\phi_{1}=\rho_{S} \alpha_{S R^{\prime}}^{2} \phi_{2}=\rho_{R} \alpha_{R F}^{2}, X=h_{S R}^{2}$ and $Y=h_{R F}^{2}$, we have

$$
\begin{aligned}
P_{\text {out }, F}^{A F} & =1-\operatorname{Pr}\left\{\frac{a_{1} \phi_{1} \phi_{2} X Y}{a_{2} \phi_{1} \phi_{2} X Y+\phi_{1} X+\phi_{2} Y+1}>\psi_{1}\right\}, \\
& =1-\operatorname{Pr}\left\{Y>\frac{\psi_{1}\left(1+\phi_{1} X\right)}{\phi_{1} \phi_{2} X\left(a_{1}-a_{2} \psi_{1}\right)-\psi_{1} \phi_{2}}\right\}, \\
& =1-\operatorname{Pr}\left\{Y>\frac{\psi_{1}\left(1+\phi_{1} x\right)}{\phi_{1} \phi_{2} x\left(a_{1}-a_{2} \psi_{1}\right)-\psi_{1} \phi_{2}} \mid X=x\right\}, \\
& =1-\int_{\Omega}^{\infty} \bar{F}_{Y}\left(\frac{\psi_{1}\left(1+\phi_{1} x\right)}{\phi_{1} \phi_{2} x\left(a_{1}-a_{2} \psi_{1}\right)-\psi_{1} \phi_{2}}\right) f_{X}(x) d x,
\end{aligned}
$$

where $f_{X}(\cdot)$ and $\bar{F}_{Y}(\cdot)$ are the PDF and CCDF of the RVs $X$ and $Y$, respectively, where $\Omega=\frac{\psi_{1} \phi_{2}}{\phi_{1} \phi_{2}\left(a_{1}-a_{2} \psi_{1}\right)}$ since $x>\left(\psi_{1} \phi_{2}\right) /\left(\phi_{1} \phi_{2}\left(a_{1}-a_{2} \psi_{1}\right)\right)$ must be satisfied. Noting that the RVs $X$ and $Y$ are log-normally distributed with parameters $h_{S R}^{2} \sim \ln \mathcal{N}\left(2 \mu_{S R}, 4 \sigma_{S R}^{2}\right)$ and $h_{R F}^{2} \sim \ln \mathcal{N}\left(2 \mu_{R F}, 4 \sigma_{R F}^{2}\right)$, respectively, we have

$$
f_{X}(x)=\frac{\xi}{x \sqrt{8 \pi} \sigma_{S R}} \exp \left[-\frac{\left(\xi \ln (x)-2 \mu_{S R}\right)^{2}}{8 \sigma_{S R}^{2}}\right]
$$

and

$$
\bar{F}_{Y}\left(\frac{\psi_{1}\left(1+\phi_{1} x\right)}{\phi_{1} \phi_{2} x\left(a_{1}-a_{2} \psi_{1}\right)-\psi_{1} \phi_{2}}\right)=Q\left(\frac{\xi \ln \left[\frac{\psi_{1}\left(1+\phi_{1} x\right)}{\phi_{1} \phi_{2} x\left(a_{1}-a_{2} \psi_{1}\right)-\psi_{1} \phi_{2}}\right]-2 \mu_{R F}}{2 \sigma_{R F}}\right) .
$$

$Q(\cdot)$ denotes the Gaussian $Q$ function defined as

$$
Q(x)=\frac{1}{\sqrt{2 \pi}} \int_{x}^{\infty} \exp \left(-\frac{t^{2}}{2}\right) d t
$$

Substituting (17) and (18) into (16), the outage probability of the far user, $\mathcal{F}$, is given by

$$
P_{o u t, F}^{A F}=\int_{\substack{\psi_{1} \phi_{2} \\ \phi_{1} \phi_{2}\left(a_{1}-a_{2} \psi_{1}\right)}}^{\infty} \frac{\xi}{x \sqrt{8 \pi} \sigma_{S R}} \exp \left[-\frac{\left(\xi \ln (x)-2 \mu_{S R}\right)^{2}}{8 \sigma_{S R}^{2}}\right] Q\left(\frac{\xi \ln \left[\frac{\psi_{1}\left(1+\phi_{1} x\right)}{\phi_{1} \phi_{2} x\left(a_{1}-a_{2} \psi_{1}\right)-\psi_{1} \phi_{2}}\right]-2 \mu_{R F}}{2 \sigma_{R F}}\right) d x,
$$

Due to SIC decoding, the outage probability of the near user $\mathcal{N}$ is expressed as [33]

$$
\begin{aligned}
P_{\text {out }, N}^{A F} & =\operatorname{Pr}\left\{\gamma_{N \rightarrow x_{1}}^{A F}<\psi_{1}, \gamma_{N \rightarrow x_{2}}^{A F}<\psi_{2}\right\}, \\
& =1-\operatorname{Pr}\left\{\gamma_{N \rightarrow x_{1}}^{A F}>\psi_{1}, \gamma_{N \rightarrow x_{2}}^{A F}>\psi_{2}\right\} .
\end{aligned}
$$

By defining $\omega_{1}=\rho_{S} \alpha_{S R}^{2}, \omega_{2}=\rho_{R} \alpha_{R N}^{2}, X=h_{S R}^{2}$ and $Z=h_{R N}^{2}$, we have 


$$
\begin{aligned}
P_{\text {out }, N}^{A F} & =1-\operatorname{Pr}\left\{\frac{a_{1} \omega_{1} \omega_{2} X Z}{a_{2} \omega_{1} \omega_{2} X Z+\omega_{1} X+\omega_{2} Z+1}>\psi_{1}, \frac{a_{2} \omega_{1} \omega_{2} X Z}{\omega_{1} X+\omega_{2} Z+1}>\psi_{2}\right\}, \\
& =1-\operatorname{Pr}\left\{Z>\frac{\psi_{1}\left(1+\omega_{1} X\right)}{\omega_{1} \omega_{2} X\left(a_{1}-a_{2} \psi_{1}\right)-\psi_{1} \omega_{2}}, Z>\frac{\psi_{2}\left(1+\omega_{1} X\right)}{a_{2} \omega_{1} \omega_{2} X-\psi_{2} \omega_{2}}\right\}, \\
& =1-\operatorname{Pr}\left\{Z>\frac{\left(1+\omega_{1} X\right)}{\left(\omega_{1} \omega_{2}\left(a_{1}-a_{2} \psi_{1}\right) X\right) /\left(\psi_{1}\right)-\omega_{2}}, Z>\frac{\left(1+\omega_{1} X\right)}{\left(a_{2} \omega_{1} \omega_{2} X\right) /\left(\psi_{2}\right)-\omega_{2}}\right\}, \\
& =1-\operatorname{Pr}\left\{Z>\frac{1+\omega_{1} x}{\Theta x-\omega_{2}} \mid X=x\right\},
\end{aligned}
$$

where

$$
\Theta=\min \left(\frac{\omega_{1} \omega_{2}\left(a_{1}-a_{2} \psi_{1}\right)}{\psi_{1}}, \frac{a_{2} \omega_{1} \omega_{2}}{\psi_{2}}\right) .
$$

The outage probability of the near user is evaluated as

$$
P_{o u t, N}^{A F}=1-\int_{\omega_{2} / \Theta}^{\infty} \bar{F}_{Z}\left(\frac{1+\omega_{1} x}{\Theta x-\omega_{2}}\right) f_{X}(x) d x
$$

where $f_{X}(\cdot)$ and $\bar{F}_{Z}(\cdot)$ are the PDF and CCDF of the RVs $X$ and $Z$, respectively. Noting that the RVs $X$ and $Z$ are log-normally distributed with parameters $h_{S R}^{2} \sim \ln \mathcal{N}\left(2 \mu_{S R}, 4 \sigma_{S R}^{2}\right)$ and $h_{R N}^{2} \sim$ $\ln \mathcal{N}\left(2 \mu_{R N}, 4 \sigma_{R N}^{2}\right)$, respectively, we have

$$
P_{o u t, N}^{A F}=\int_{\omega_{2} / \Theta}^{\infty} \frac{\xi}{x \sqrt{8 \pi} \sigma_{S R}} \exp \left[-\frac{\left(\xi \ln (x)-2 \mu_{S R}\right)^{2}}{8 \sigma_{S R}^{2}}\right] Q\left(\frac{\xi \ln \left[\frac{1+\omega_{1} x}{\Theta x-\omega_{2}}\right]-2 \mu_{R N}}{2 \sigma_{R N}}\right) d x,
$$

where $\Theta x-\omega_{2}>0$ must be satisfied.

\subsection{Asymptotic Outage Probability of AF NOMA}

In this section, we analyze the user outage probabilities of the AF NOMA at high SNR. This allows us to derive closed-form lower and upper bounds of the exact outage probabilities of the far user and the near user obtained in (20) and (25), respectively. Through simulation results, it will be shown that the lower bounds are tight particularly at high SNR. First, we assume equal source and relay transmit SNR such that $\rho=\rho_{S}=\rho_{R}$. Using (7), (8) and (9), the outage probabilities of the far user and the near user are, respectively, re-expressed as [14]

$$
P_{o u t, F}^{A F}=1-\operatorname{Pr}\left\{\frac{\rho \alpha_{S R}^{2} X Y}{\rho \alpha_{S R}^{2} X+\rho \alpha_{R F}^{2} Y+1}>\delta_{F}\right\}
$$

and

$$
P_{o u t, N}^{A F}=1-\operatorname{Pr}\left\{\frac{\rho \alpha_{S R}^{2} X Z}{\rho \alpha_{S R}^{2} X+\rho \alpha_{R N}^{2} Z+1}>\delta_{N}\right\}
$$

where

$$
\delta_{F}=\frac{\psi_{1}}{\rho \alpha_{R F}^{2}\left(a_{1}-\psi_{1} a_{2}\right)}
$$


and

$$
\delta_{N}=\frac{\psi_{2}}{a_{2} \rho \alpha_{R N}^{2}} .
$$

In the high SNR region (i.e., $\rho \rightarrow \infty$ ) and from (26), the outage probability of the far user is given by

$$
P_{\text {out }, F}^{A F} \stackrel{(a)}{=} 1-\operatorname{Pr}\left\{\frac{\mathcal{X} Y}{\mathcal{X}+Y}>\delta_{F}\right\}
$$

where $(a)$ is obtained by substituting $\mathcal{X}=\kappa_{F} X$ and $\kappa_{F}=\alpha_{S R}^{2} / \alpha_{R F}^{2} \cdot \mathcal{X}$ is a log-normal RV distributed as $\mathcal{X} \sim \ln \mathcal{N}\left(2 \mu_{S R}+\xi \ln \left(\kappa_{F}\right), 4 \sigma_{S R}^{2}\right)$. According to Reference [14], the following inequality holds

$$
\frac{1}{2} \min (u, v) \leq \frac{u v}{u+v} \leq \min (u, v),
$$

where $u$ and $v$ are RVs. Based on (31), the lower bound (LB) and upper bound (UB) for the outage probability of the far user are, respectively, found as

$$
\begin{aligned}
P_{o u t, F}^{A F, L B} & =1-\operatorname{Pr}\left\{\min (\mathcal{X}, Y)>\delta_{F}\right\}, \\
& =F_{\mathcal{X}}\left(\delta_{F}\right)+F_{Y}\left(\delta_{F}\right)-F_{\mathcal{X}}\left(\delta_{F}\right) F_{Y}\left(\delta_{F}\right),
\end{aligned}
$$

and

$$
\begin{aligned}
P_{\text {out }, F}^{A F, U B} & =1-\operatorname{Pr}\left\{\min (\mathcal{X}, Y)>2 \delta_{F}\right\}, \\
& =F_{\mathcal{X}}\left(2 \delta_{F}\right)+F_{Y}\left(2 \delta_{F}\right)-F_{\mathcal{X}}\left(2 \delta_{F}\right) F_{Y}\left(2 \delta_{F}\right) .
\end{aligned}
$$

The $\mathrm{CDFs} F_{\mathcal{X}}(\cdot)$ and $F_{Y}(\cdot)$ are, respectively, given by

$$
F_{\mathcal{X}}(\eta)=1-Q\left(\frac{\xi \ln [\eta]-2 \mu_{S R}-\xi \ln \left[\kappa_{F}\right]}{2 \sigma_{S R}}\right),
$$

and

$$
F_{Y}(\eta)=1-Q\left(\frac{\xi \ln [\eta]-2 \mu_{R F}}{2 \sigma_{R F}}\right),
$$

where $\eta=\left\{\delta_{F}, 2 \delta_{F}\right\}$ for $\{L B, U B\}$, respectively. By following a similar procedure as above, the LB and UB for the outage probability of the near user are, respectively, represented as

$$
P_{o u t, N}^{A F, L B}=F_{\mathcal{W}}\left(\delta_{N}\right)+F_{Z}\left(\delta_{N}\right)-F_{\mathcal{W}}\left(\delta_{N}\right) F_{Z}\left(\delta_{N}\right),
$$

and

$$
P_{\text {out }, N}^{A F, U B}=F_{\mathcal{W}}\left(2 \delta_{N}\right)+F_{Z}\left(2 \delta_{N}\right)-F_{\mathcal{W}}\left(2 \delta_{N}\right) F_{Z}\left(2 \delta_{N}\right)
$$

The $\mathrm{CDFs} F_{\mathcal{W}}(\cdot)$ and $F_{Z}(\cdot)$ are, respectively, expressed by

$$
F_{\mathcal{W}}(\lambda)=1-Q\left(\frac{\xi \ln [\lambda]-2 \mu_{S R}-\xi \ln \left[\kappa_{N}\right]}{2 \sigma_{S R}}\right),
$$

and

$$
F_{Z}(\lambda)=1-Q\left(\frac{\xi \ln [\lambda]-2 \mu_{R N}}{2 \sigma_{R N}}\right)
$$


where $\lambda=\left\{\delta_{N}, 2 \delta_{N}\right\}$ for $\{L B, U B\}$, respectively. $\mathcal{W}$ is a log-normal RV distributed as $\mathcal{W} \sim$ $\ln \mathcal{N}\left(2 \mu_{S R}+\xi \ln \left(\kappa_{N}\right), 4 \sigma_{S R}^{2}\right)$ and $\kappa_{N}=\alpha_{S R}^{2} / \alpha_{R N}^{2}$.

\subsection{Decode-and-Forward NOMA}

Based on (10) and (12), the outage probability of the far user, $\mathcal{F}$, is expressed as

$$
P_{\text {out }, F}^{D F}=1-\underbrace{\operatorname{Pr}\left\{\gamma_{R \rightarrow x_{1}}^{D F}>\psi_{1}\right\}}_{\mathcal{J}_{1}} \underbrace{\operatorname{Pr}\left\{\gamma_{F \rightarrow x_{1}}^{D F}>\psi_{1}\right\}}_{\mathcal{J}_{2}} .
$$

$\mathcal{J}_{1}$ is evaluated as

$$
\begin{aligned}
\mathcal{J}_{1} & =\operatorname{Pr}\left\{\gamma_{R \rightarrow x_{1}}^{D F}>\psi_{1}\right\} \\
& =\operatorname{Pr}\left\{\frac{a_{1} \rho_{S} \alpha_{S R}^{2} h_{S R}^{2}}{a_{2} \rho_{S} \alpha_{S R}^{2} h_{S R}^{2}+1}>\psi_{1}\right\} \\
& =\operatorname{Pr}\left\{h_{S R}^{2}>\frac{\psi_{1}}{\rho_{S} \alpha_{S R}^{2}\left(a_{1}-\psi_{1} a_{2}\right)}\right\} \\
& =Q\left(\frac{\xi \ln \left[\psi_{1}\right]-2 \mu_{S R}-\xi \ln \left[\rho_{S} \alpha_{S R}^{2}\left(a_{1}-\psi_{1} a_{2}\right)\right]}{2 \sigma_{S R}}\right),
\end{aligned}
$$

where $a_{1}-\psi_{1} a_{2}>0$ must be satisfied. Similarly, using (12), $\mathcal{J}_{2}$ is calculated as

$$
\mathcal{J}_{2}=Q\left(\frac{\xi \ln \left[\psi_{1}\right]-2 \mu_{R F}-\xi \ln \left[\rho_{R} \alpha_{R F}^{2}\left(a_{1}-\psi_{1} a_{2}\right)\right]}{2 \sigma_{R F}}\right) .
$$

By substituting (41) and (42) into (40), the outage probability of the far user with DF NOMA is given by

$$
P_{\text {out }, F}^{D F}=1-Q\left(\frac{\xi \ln \left[\frac{\psi_{1}}{\rho_{S} \alpha_{S R}^{2}\left(a_{1}-\psi_{1} a_{2}\right)}\right]-2 \mu_{S R}}{2 \sigma_{S R}}\right) Q\left(\frac{\xi \ln \left[\frac{\psi_{1}}{\rho_{R} \alpha_{R F}^{2}\left(a_{1}-\psi_{1} a_{2}\right)}\right]-2 \mu_{R F}}{2 \sigma_{R F}}\right) .
$$

Based on (10), (11), (13) and (14), the outage probability of the near user, $\mathcal{N}$, is given by [16]

$$
P_{o u t, N}^{D F}=1-\underbrace{\operatorname{Pr}\left\{\gamma_{R \rightarrow x_{1}}^{D F}>\psi_{1}, \gamma_{R \rightarrow x_{2}}^{D F}>\psi_{2}\right\}}_{\mathcal{J}_{1}} \underbrace{\operatorname{Pr}\left\{\gamma_{N \rightarrow x_{1}}^{D F}>\psi_{1}, \gamma_{N \rightarrow x_{2}}^{D F}>\psi_{2}\right\}}_{\mathcal{J}_{2}} .
$$

Here, $\mathcal{J}_{1}$ is calculated as

$$
\begin{aligned}
\mathcal{J}_{1} & =\operatorname{Pr}\left\{\gamma_{R \rightarrow x_{1}}^{D F}>\psi_{1}, \gamma_{R \rightarrow x_{2}}^{D F}>\psi_{2}\right\}, \\
& =\operatorname{Pr}\left\{\frac{a_{1} \rho_{S} \alpha_{S R}^{2} h_{S R}^{2}}{a_{2} \rho_{S} \alpha_{S R}^{2} h_{S R}^{2}+1}>\psi_{1}, a_{2} \rho_{S} \alpha_{S R}^{2} h_{S R}^{2}>\psi_{2}\right\}, \\
& =\operatorname{Pr}\left\{h_{S R}^{2}>\frac{\psi_{1}}{\rho_{S} \alpha_{S R}^{2}\left(a_{1}-\psi_{1} a_{2}\right)}, h_{S R}^{2}>\frac{\psi_{2}}{a_{2} \rho_{S} \alpha_{S R}^{2}}\right\}, \\
& =Q\left(\frac{\xi \ln (\Phi)-2 \mu_{S R}}{2 \sigma_{S R}}\right),
\end{aligned}
$$


where

$$
\Phi=\max \left(\frac{\psi_{1}}{\rho_{S} \alpha_{S R}^{2}\left(a_{1}-\psi_{1} a_{2}\right)}, \frac{\psi_{2}}{a_{2} \rho_{S} \alpha_{S R}^{2}}\right) .
$$

Following $\mathcal{J}_{1}, \mathcal{J}_{2}$ is derived as

$$
\mathcal{J}_{2}=Q\left(\frac{\xi \ln (\Xi)-2 \mu_{R N}}{2 \sigma_{R N}}\right)
$$

where

$$
\Xi=\max \left(\frac{\psi_{1}}{\rho_{R} \alpha_{R N}^{2}\left(a_{1}-\psi_{1} a_{2}\right)}, \frac{\psi_{2}}{a_{2} \rho_{R} \alpha_{R N}^{2}}\right)
$$

By substituting (45) and (47) into (44), the outage probability of the far user with DF NOMA is expressed as

$$
P_{\text {out }, N}^{D F}=1-Q\left(\frac{\xi \ln (\Phi)-2 \mu_{S R}}{2 \sigma_{S R}}\right) Q\left(\frac{\xi \ln (\Xi)-2 \mu_{R N}}{2 \sigma_{R N}}\right)
$$

\subsection{System Throughput}

Subsequently, we analyze the system throughput performance. When the system transmits at a fixed data rate, the throughput is an important metric to characterize system performance. The throughput is the product of the fixed transmission rate and the successful communication probability [13]. The system throughput, $\tau_{\text {sum }}$, of the proposed NOMA PLC network is deduced as

$$
\begin{aligned}
\tau_{\text {sum }}^{j} & =\tau_{F}^{j}+\tau_{N^{\prime}}^{j} \\
& =R_{1}\left(1-P_{o u t, F}^{j}\right)+R_{2}\left(1-P_{o u t, N}^{j}\right),
\end{aligned}
$$

where $j \in\{A F, D F\} . \tau_{F}^{j}$ and $\tau_{N}^{j}$ are the throughputs of the far user and the near user, respectively. For AF NOMA, $P_{\text {out }, F}^{A F}$ and $P_{o u t, N}^{A F}$ are obtained from (20) and (25), respectively. For DF NOMA, $P_{\text {out }, F}^{D F}$ and $P_{o u t, N}^{D F}$ are obtained from (43) and (49), respectively.

Optimum power allocation is essential to maximizing the system throughput of the proposed cooperative NOMA schemes. However, due to the complicated form of the outage probability expressions, it is very difficult to obtain closed-from expressions for the power allocation parameters. In this case, maximum system throughput is derived by performing an exhaustive search over the range of power allocation coefficients. To maximize the system throughput, the power allocation problem is formulated as [34]

$$
\begin{array}{ll}
\underset{a_{1}}{\operatorname{maximize}} & \tau_{\text {sum }}\left(a_{1}, a_{2}, \alpha_{i}^{2}, \sigma_{i}^{2}, \mu_{i}, d_{i}, p, \rho_{S}, \rho_{R}\right) \\
\text { subject to } & 0<a_{1}<1
\end{array}
$$

\section{Benchmark Schemes}

To highlight the performance of the AF and DF NOMA schemes under various settings, this section presents two different benchmark schemes namely NOMA without relaying and OMA schemes, respectively.

\subsection{NOMA without Relaying (D-NOMA)}

The NOMA without relaying scheme is used as a benchmark for comparison. In the D-NOMA transmission, the source transmits the data signals $x_{1}$ and $x_{2}$ with appropriate power allocation 
directly to the designated users without the help of the relay. In line with the NOMA protocol with SIC, the users can recover their desired signals.

\subsection{OMA Transmission}

As a benchmark scheme for comparison, the source sends information to the two users via the relay using a OMA scheme [35]. The information transfer is completed in four orthogonal phases. The source transmit their data signals $x_{1}$ and $x_{2}$ to the relay in the first and second phases, respectively. In the third and fourth phases, the relay forwards the data signals $x_{1}$ and $x_{2}$ to the far user and the near user, respectively. Each data signal is transmitted at full power.

\section{Simulation Results}

In this section, we present extensive simulation results to evaluate the performance of the proposed cooperative NOMA schemes in PLC networks. Our results are validated through Monte Carlo simulations averaged over $10^{5}$ channel realizations. To characterize the power line attenuation, we adopt the following parameters: $b_{0}=9.4 \times 10^{-3}, b_{1}=4.2 \times 10^{-7}, k=0.7$ and $f=30 \mathrm{MHz}$ [9]. The power allocation coefficients of the NOMA transmission are fixed such that $a_{1}=0.75$ and $a_{2}=0.25$ [19-21]. For simplicity, we consider equal channel mean and variances such that $\mu_{S R}=\mu_{R N}=\mu_{R F}=3 \mathrm{~dB}$ and $\sigma_{S R}^{2}=\sigma_{R N}^{2}=\sigma_{R F}^{2}=4 \mathrm{~dB}$ [36]. The probability of impulsive noise occurrence is set as $p=0.01$ and the target rates are given by $R_{1}=0.5 \mathrm{bps} / \mathrm{Hz}$ and $R_{2}=1 \mathrm{bps} / \mathrm{Hz}$, respectively. Unless otherwise stated, $d_{S R}=200 \mathrm{~m}, d_{R N}=100 \mathrm{~m}$ and $d_{R F}=200 \mathrm{~m}$, respectively.

In Figure 2, we plot the outage probability of the AF NOMA scheme versus the source transmit SNR. We illustrate the performance for a fixed power allocation and equal source and relay transmit powers that is, $\rho_{S}=\rho_{R}$. From the plot, we can observe that the analytic results show a tight approximation to the simulation results verifying the accuracy of the derived analytic results in (20) and (25). Also, the derived closed-form LBs of the outage probability for the far and near users obtained from (32) and (36), respectively are very tight especially at high SNR. Therefore, they can be used to approximate the system performance.

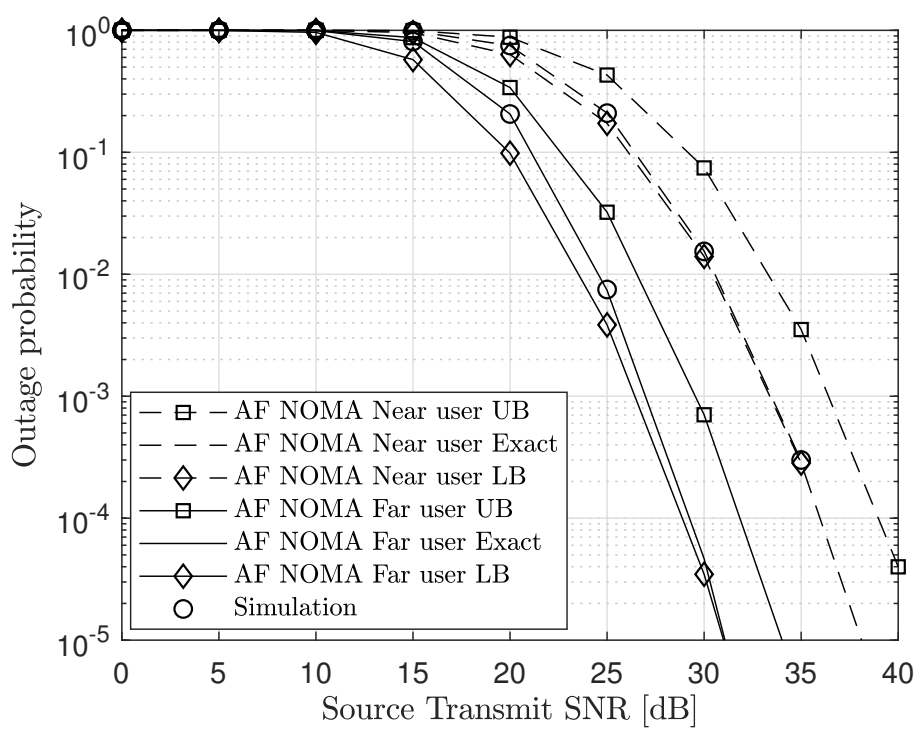

Figure 2. Outage probability versus source transmit SNR for $p=0.01, a_{1}=0.75, a_{2}=0.25$, $R_{1}=0.5 \mathrm{bps} / \mathrm{Hz}$ and $R_{2}=1 \mathrm{bps} / \mathrm{Hz}$.

Figure 3 shows the outage probability performance versus the source transmit SNR for the different schemes. We compare the DF and AF NOMA schemes to the benchmark schemes presented in Section 4. From the plot, we can observe that the analytic results show a tight approximation to the 
simulation results verifying the accuracy of the derived analytic results. For the near and far users, the outage probability of the proposed AF and DF NOMA schemes are enhanced compared to the OMA and NOMA without relaying schemes as shown in Figure $3 \mathrm{a}, \mathrm{b}$, respectively. By allocating more power to the far user, the AF and DF NOMA schemes are able to ensure user fairness. Therefore, multiple users can be served concurrently while achieving set quality of service (QoS) requirements. From Figure 3a, it is seen that the DF NOMA scheme has better outage probability than the AF NOMA when the channel variance is low (i.e., $\sigma_{S R}^{2}=\sigma_{R N}^{2}=\sigma_{R F}^{2}=4 \mathrm{~dB}$ ). However, as the channel variance increases from $4 \mathrm{~dB}$ to $10 \mathrm{~dB}$, the outage probability is similar for the AF and DF NOMA schemes across the whole SNR range. In essence, while DF NOMA scheme can be chosen as the preferred protocol when the channel conditions are good, the AF NOMA scheme can be selected as more loads are connected to network due to its low computational complexity. For the near user, the performance of the AF and DF NOMA schemes remains similar in low and high channel variance settings. As the NOMA without relaying scheme suffers from high signal attenuation, it has the worst outage probability performance.

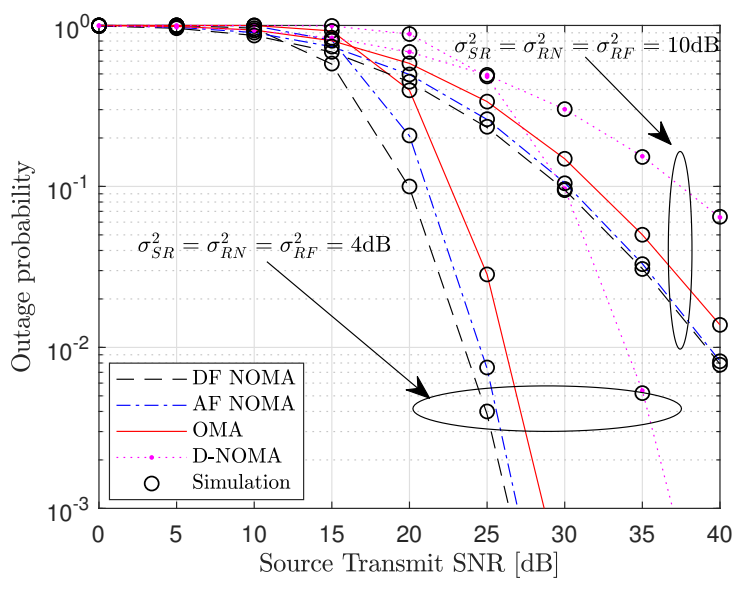

(a)

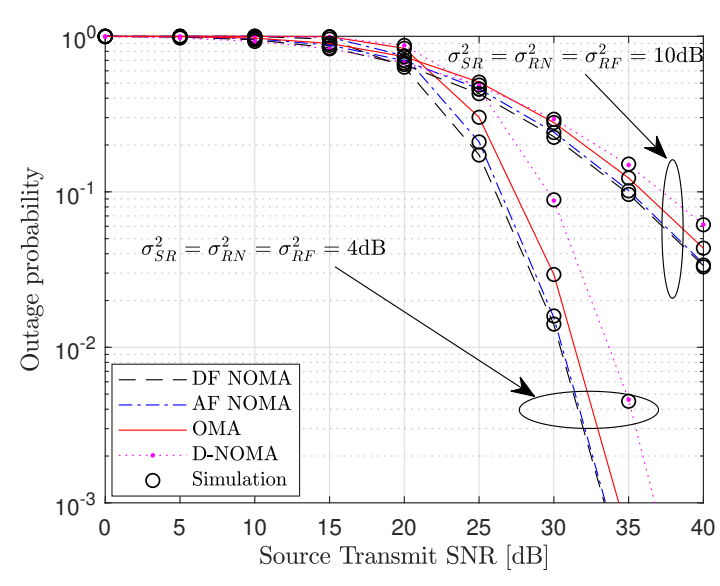

(b)

Figure 3. Outage probability performance of different schemes for (a) far user and (b) near user where $p=0.01, a_{1}=0.75, a_{2}=0.25, R_{1}=0.5 \mathrm{bps} / \mathrm{Hz}$ and $R_{2}=1 \mathrm{bps} / \mathrm{Hz}$.

Figure 4 shows the system throughput versus the source transmit SNR. The system throughput is plotted using (50). It is observed that the proposed DF and AF NOMA schemes significantly enhance the system throughput relative to the benchmark schemes. The system throughput is enhanced when the relay employs DF instead of AF protocol. This follows directly from the outage probability performance of the two schemes. The AF and DF NOMA schemes will require less power to achieve a set target rate compared to the OMA scheme. For example, for a target rate of $0.5 \mathrm{bps} / \mathrm{Hz}$ and $p=0.01$, the DF and AF NOMA schemes will require $17.5 \mathrm{~dB}$ and $18 \mathrm{~dB}$, respectively while the OMA scheme will require $20 \mathrm{~dB}$. Finally, we observe that when the impulsive noise probability increases from $p=0.01$ to $p=0.2$, the outage probability performance is degraded across all schemes. This is because higher $p$ means more received samples are corrupted by impulsive noise and discarded in the decoding process.

Figure 5 depicts the maximum system throughput versus the far user target rate, $R_{1}$. The maximum system throughput is obtained from (51). Specifically, we set the following parameters: $\rho_{S}=30 \mathrm{~dB}, \rho_{R}=20 \mathrm{~dB}$ and $p=0.01$. From the results, it is shown that the DF NOMA scheme has the best performance while the OMA scheme has the worst performance. The gap between the NOMA schemes and the OMA scheme increases as $R_{2}$ increases. However, the system throughput of the $\mathrm{DF}$ and AF NOMA schemes is dependent on the given target rates of the users. At $R_{2}=1 \mathrm{bps} / \mathrm{Hz}$, as the target rate of the far user $R_{1}$ increases from $0.2 \mathrm{bps} / \mathrm{Hz}$ to $0.9 \mathrm{bps} / \mathrm{Hz}$, the throughput of the DF NOMA scheme is enhanced compared to the NOMA without relaying scheme. Beyond $0.9 \mathrm{bps} / \mathrm{Hz}$, 
the DF NOMA scheme fails to guarantee the system QoS, hence the system throughput is degraded. A similar observation is made for the AF NOMA scheme where the NOMA without relaying scheme begins to outperform the AF NOMA scheme when $R_{1}>0.6 \mathrm{bps} / \mathrm{Hz}$. Therefore target rates need to be carefully selected for the AF and DF NOMA schemes to outperform the benchmark schemes.

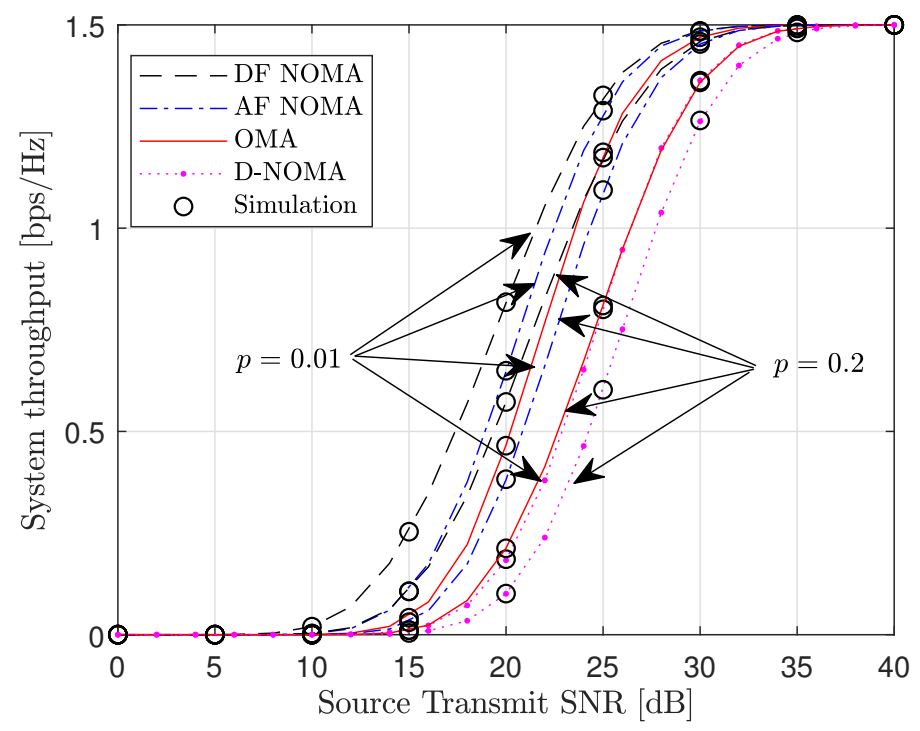

Figure 4. System throughput versus the source transmit SNR for $a_{1}=0.75, a_{2}=0.25, R_{1}=0.5 \mathrm{bps} / \mathrm{Hz}$ and $R_{2}=1 \mathrm{bps} / \mathrm{Hz}$.

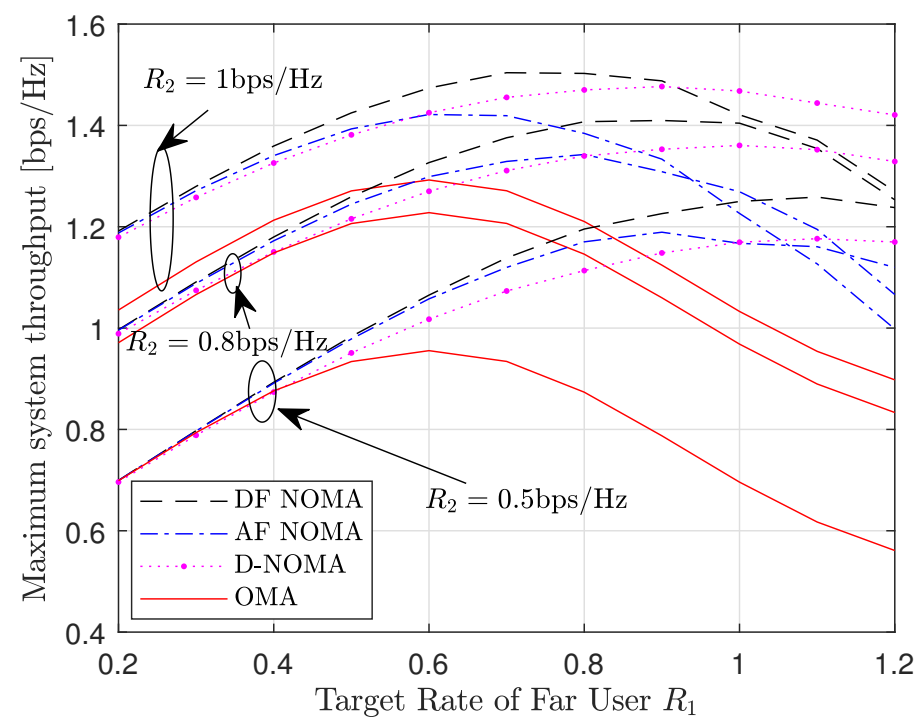

Figure 5. Maximum system throughput versus the far user target rate for $p=0.01, \rho_{S}=30 \mathrm{~dB}$ and $\rho_{R}=20 \mathrm{~dB}$.

Finally, Figure 6 examines the impact of the relay position on the performance of the proposed NOMA schemes. Specifically, we plot the maximum system throughput versus the source-to-relay distance $d_{S R}$. The maximum system throughput is plotted using (51). We assume the source-to-far user distance is $500 \mathrm{~m}$ and the near user is located $100 \mathrm{~m}$ from the far user. From the results, there exists an optimum relay position that maximizes the system throughput. Although the received signal power is high when the relay is close to the source, the far distance between the relay and the users means the forwarded signal in the second phase is highly attenuated, degrading the system throughput. For a fixed relay power, the system throughput is enhanced when the source transmits with more 
power. In addition, we observe that the DF NOMA scheme is able to enhance the system throughput compared to the other schemes for all relay positions.

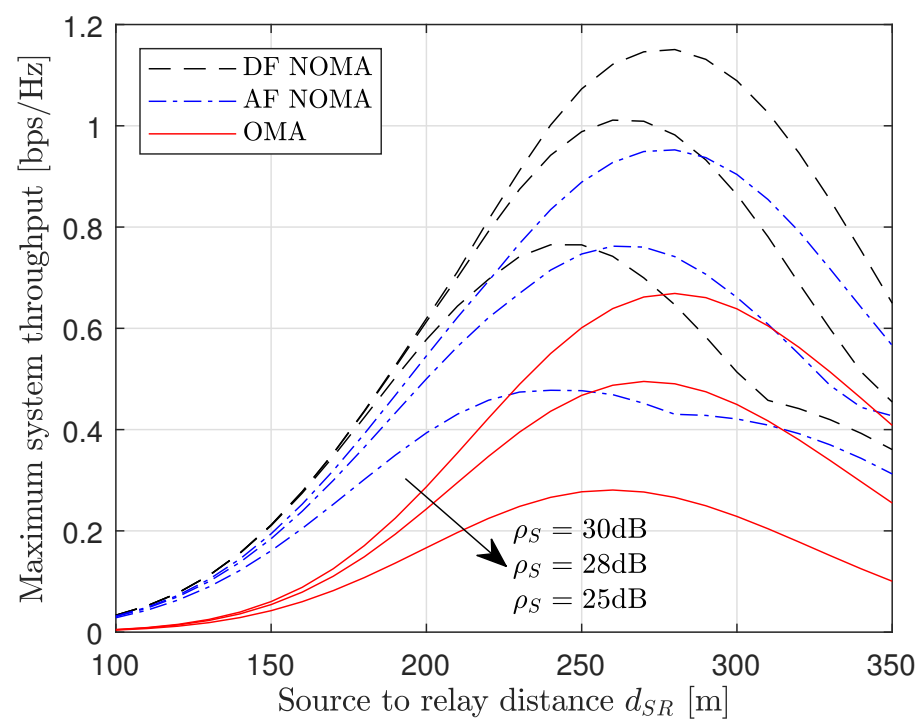

Figure 6. Maximum system throughput versus source-to-relay distance $d_{S R}$ for for $p=0.01, \rho_{R}=20 \mathrm{~dB}$ and $R_{1}=0.5 \mathrm{bps} / \mathrm{Hz}$ and $R_{2}=1 \mathrm{bps} / \mathrm{Hz}$.

\section{Conclusions}

In this paper, we have proposed cooperative NOMA for PLC networks where the relay aids information transfer between the source and the users. The source uses superposition coding for data transmission to the relay. The relay uses AF or DF protocol and forwards the received signal to the users. We derived analytic expressions for the outage probability and the throughput under PLC log-normal channels with impulsive noise. For AF NOMA scheme, we analyzed the results at high SNR and derived closed-form lower and upper bounds for the outage probability. Through Monte Carlo simulations, we have verified the accuracy of the analysis. The simulation results revealed the superiority of the cooperative NOMA schemes compared to the benchmark schemes in all performance metrics. Comparing AF and DF NOMA, it was shown that DF NOMA has better outage probability than AF NOMA especially when the channel variance is low. Furthermore, the DF NOMA scheme achieves a higher system throughput compared to the AF NOMA scheme. Due to synchronization and channel estimation issues, imperfect SIC can be considered as an extension to this work.

Author Contributions: Conceptualization, Methodology, and Original Draft Preparation R.K.A.; Writing一review and editing, Validation P.A.; Supervision, K.-J.L.

Funding: This research was supported by the research fund of Hanbat National University in 2019.

Conflicts of Interest: The authors declare no conflict of interest.

\section{References}

1. Ding, Z.; Lei, X.; Karagiannidis, G.K.; Schober, R.; Yuan, J.; Bhargava, V.K. A Survey on Non-Orthogonal Multiple Access for 5G Networks: Research Challenges and Future Trends. IEEE J. Sel. Areas Commun. 2017, 35, 2181-2195. [CrossRef]

2. Choi, J. On Generalized Downlink Beamforming with NOMA. J. Commun. Netw. 2017, 19, 319-328. [CrossRef]

3. Zeng, M.; Yadav, A.; Dobre, O.A.; Tsiropoulos, G.I.; Poor, H.V. Capacity Comparison between MIMO-NOMA and MIMO-OMA with Multiple Users in a Cluster. IEEE J. Sel. Areas Commun. 2017, 35, $2413-2424$. [CrossRef] 
4. Higuchi, K.; Benjebbour, A. Non-orthogonal Multiple Access (NOMA) with Successive Interference Cancellation for Future Radio Access. IEICE Trans. Commun. 2015, 98-B, 403-414. [CrossRef]

5. Lee, K.-J.; Kim, J.-S.; Caire, G.; Lee, I. Asymptotic Ergodic Capacity Analysis for MIMO Amplify-and-Forward Relay Networks. IEEE Trans. Wireless Commun. 2010, 9, 2712-2717. [CrossRef]

6. Song, C.; Lee, K.-J.; Lee, I. MMSE-Based MIMO Cooperative Relaying Systems: Closed-Form Designs and Outage Behavior. IEEE J. Sel. Areas Commun. 2012, 30, 1390-1401. [CrossRef]

7. Park, J.; Chae, C.-B.; Yoon, G. Amplify-and-Forward Two-Way Relaying System over Free-Space Optics Channels. J. Commun. Netw. 2017, 19, 481-492. [CrossRef]

8. Asiedu, D.K.P.; Mahama, S.; Jeon, S.-W.; Lee, K.-J. Optimal Power Splitting for Simultaneous Wireless Information and Power Transfer in Amplify-and-Forward Multiple-Relay Systems. IEEE Access 2018, 6, 3459-3468. [CrossRef]

9. Ahiadormey, R.K.; Anokye, P.; Jo, H.-S.; Lee, K.-J. Performance Analysis of Two-Way Relaying in Cooperative Power Line Communications. IEEE Access 2019, 7, 97264-97280. [CrossRef]

10. Yu, M.; Li, J. Is Amplify-and-Forward Practically Better Than Decode-and-Forward or Vice Versa? In Proceedings of the IEEE International Conference on Acoustics, Speech, and Signal Processing (ICASSP), Philadelphia, PA, USA, 23 March 2005; Volume 3, pp. 365-368. [CrossRef]

11. Kim, J.-B.; Lee, I.-H. Capacity Analysis of Cooperative Relaying Systems Using Non-Orthogonal Multiple Access. IEEE Commun. Lett. 2015, 19, 1949-1952. [CrossRef]

12. Xu, M.; Ji, F.; Wen, M.; Duan, W. Novel Receiver Design for the Cooperative Relaying System With Non-Orthogonal Multiple Access. IEEE Commun. Lett. 2016, 20, 1679-1682. [CrossRef]

13. Yue, X.; Liu, Y.; Kang, S.; Nallanathan, A.; Ding, Z. Exploiting Full/Half-Duplex User Relaying in NOMA Systems. IEEE Trans. Commun. 2018, 66, 560-575. [CrossRef]

14. Men, J.; Ge, J.; Zhang, C. Performance Analysis of Nonorthogonal Multiple Access for Relaying Networks Over Nakagami-m Fading Channels. IEEE Trans. Veh. Technol. 2017, 66, 1200-1208. [CrossRef]

15. Yue, X.; Liu, Y.; Kang, S.; Nallanathan, A. Performance Analysis of NOMA with Fixed Gain Relaying over Nakagami- $m$ Fading Channels. IEEE Access 2017, 5, 5445-5454. [CrossRef]

16. Wan, D.; Wen, M.; Ji, F.; Liu, Y.; Huang, Y. Cooperative NOMA Systems with Partial Channel State Information Over Nakagami- $m$ Fading Channels. IEEE Trans. Commun. 2018, 66, 947-958. [CrossRef]

17. Kader, M.F.; Shahab, M.B.; Shin, S.Y. Exploiting Non-Orthogonal Multiple Access in Cooperative Relay Sharing. IEEE Commun. Lett. 2017, 21, 1159-1162. [CrossRef]

18. Nikfar, B.; Han Vinck, A.J. Relay Selection in Cooperative Power Line Communication: A Multi-Armed Bandit Approach. J. Commun. Netw. 2017, 19, 1-9. [CrossRef]

19. Rabie, K.M.; Adebisi, B.; Yousif, E.H.G.; Gacanin, H.; Tonello, A.M. A Comparison between Orthogonal and Non-Orthogonal Multiple Access in Cooperative Relaying Power Line Communication Systems. IEEE Access 2017, 5, 10118-10129. [CrossRef]

20. Rabie, K.M.; Adebisi, B.; Tonello, A.M.; Yarkan, S.; Ijaz, M. Two-Stage Non-Orthogonal Multiple Access over Power Line Communication Channels. IEEE Access 2018, 6, 17368-17376. [CrossRef]

21. Pu, H.; Liu, X.; Zhang, S.; Xu, D. Adaptive Cooperative Non-Orthogonal Multiple Access-Based Power Line Communication. IEEE Access 2019, 7, 73856-73869. [CrossRef]

22. Feng, S.; Bai, T.; Hanzo, L. Joint Power Allocation for the Multi-User NOMA-Downlink in a Power-Line-Fed VLC Network. IEEE Trans. Veh. Technol. 2019, 68, 5185-5190. [CrossRef]

23. Girotto, M.; Tonello, A.M. EMC Regulations and Spectral Constraints for Multicarrier Modulation in PLC. IEEE Access 2017, 5, 4954-4966. [CrossRef]

24. Yu, Z.; Zhai, C.; Liu, J.; Xu, H. Cooperative Relaying Based Non-Orthogonal Multiple Access (NOMA) With Relay Selection. IEEE Trans. Veh. Technol. 2018, 67, 11606-11618. [CrossRef]

25. Versolatto, F.; Tonello, A.M. Analysis of the PLC Channel Statistics using a Bottom-Up Random Simulator. In Proceedings of the IEEE International Symposium on Power Line Communications and Its Applications (ISPLC), Rio de Janeiro, Brazil, 28-31 March 2010; pp. 236-241. [CrossRef]

26. Rabie, K.M.; Adebisi, B.; Gacanin, H.; Nauryzbayev, G.; Ikpehai, A. Performance Evaluation of Multi-Hop Relaying over Non-Gaussian PLC channels. J. Commun. Netw. 2017, 19, 531-538. [CrossRef]

27. Qian, Y.; Yan, J.; Guan, H.; Li, J.; Zhou, X.; Guo, S.; Jayakody, D.N.K. Design of Hybrid Wireless and Power Line Sensor Networks with Dual-Interface Relay in IoT. IEEE Internet Things J. 2019, 6, 239-249. [CrossRef] 
28. Shongwey, T.; Han Vinck, A.J.; Ferreira, H.C. On Impulse Noise and Its Models. In Proceedings of the IEEE International Symposium on Power Line Communications and Its Applications (ISPLC), Glasgow, UK, 30 March-2 April 2014; pp. 12-17. [CrossRef]

29. Han, B.; Stoica, V.; Kaiser, C.; Otterbach, N.; Dostert, K. Noise Characterization and Emulation for Low-Voltage Power Line Channels across Narrowband and Broadband. Digit. Signal Process. 2017, 69, 259-274. [CrossRef]

30. Zimmermann, M.; Dostert, K. A Multipath Model for the Powerline Channel. IEEE Trans. Commun. 2002, 50, 553-559. [CrossRef]

31. Qian, Y.; Tian, M.; Jiang, X.; Song, H.; Shu, F.; Li, J. Performance analysis for a two-way relaying power line network with analog network coding. Front. Inf. Technol. Electron. Eng. 2015, 16, 892-898. [CrossRef]

32. Meng, H.; Guan, Y.L.; Chen, S. Modeling and Analysis of Noise Effects on Broadband Power-line Communications. IEEE Trans. Power Deliv. 2005, 20, 630-637. [CrossRef]

33. Do, D.T.; Nguyen, T.T.T. Impacts of Imperfect SIC and Imperfect Hardware in Performance Analysis on AF Non-Orthogonal Multiple Access Network. Telecommun. Syst. 2019. [CrossRef]

34. Rabie, K.M.; Adebisi, B. Enhanced Amplify-and-Forward Relaying in Non-Gaussian PLC Networks. IEEE Access 2017, 5, 4087-4094. [CrossRef]

35. Wang, Z.; Peng, Z. Secrecy Performance Analysis of Relay Selection in Cooperative NOMA Systems. IEEE Access 2019, 7, 86274-86287. [CrossRef]

36. Atallah, M.; Kaddoum, G. Design and Performance Analysis of Secure Multicasting Cooperative Protocol for Wireless Sensor Network Applications. IEEE Wirel. Commun. Lett. 2019. [CrossRef]

(C) 2019 by the authors. Licensee MDPI, Basel, Switzerland. This article is an open access article distributed under the terms and conditions of the Creative Commons Attribution (CC BY) license (http:// creativecommons.org/licenses/by/4.0/). 